\title{
The Information Integrity Imperative
}

\author{
Madhavan K. Nayar \\ Unitech Systems Inc. \\ 1240 East Diehl Road \\ Suite 300, Naperville, IL 60653-1439, USA \\ mnayar@unitechsys.com
}

Abstract: This white paper examines several dimensions of information integrity. It then
explains why it is so imperative that we mobilize appropriate resources to
create and implement a new, universal framework for achieving information
integrity.

Key words: information integrity, data quality, imperative, pervasive, information integrity space

\section{INTRODUCTION}

Information integrity is the dependability or trustworthiness of information. More specifically, in the context of today's information systems, it is the accuracy, consistency and reliability of the information content, process and system.

Every organization in business, government and society is concerned about information integrity and impacted by information integrity failures. Until now, though, the issue of information integrity has not been recognized as a pervasive, universal phenomenon, despite the fact that it costs the economy hundreds of billions of dollars. This situation offers opportunities for research, education and improvement.

In the past, we have been able to achieve an acceptable level of information integrity because we either had the time to detect and correct 
information integrity failures or the failures were sufficiently isolated to prevent wide-scale contamination (due to the loosely coupled or discontinuous nature of our systems and processes). However, several recent trends in information technology and the business environment are dramatically raising the seriousness of the problem and the urgency of finding radically different approaches and solutions for achieving information integrity.

\section{DATA QUALITY VERSUS INFORMATION INTEGRITY}

Many organizations have been engaged in major initiatives to build data warehouses of customer information. "Data quality" has become part of the vernacular of the information systems professionals involved in these initiatives. They also use such terms as "Data integrity", "Information quality", "Information integrity", "Data accuracy" and "Information accuracy" to refer to various aspects of the usefulness, usability and integrity of data and information. In this document, we use the term "Information integrity" to describe the accuracy, consistency and reliability of the information content, processes and systems. In this sense, information integrity is the basis and prerequisite for the usefulness and usability of information. It is a specific, objective attribute that lends itself to standards, measurement and improvement.

\section{THE INFORMATION INTEGRITY SPACE}

Conceptually, information integrity can be viewed and understood from many different perspectives or dimensions, ranging from purely technical issues such as the accuracy of prices recorded by a supermarket scanner to more philosophical issues such as the integrity of financial reports when they affect personal gains or losses through incentive plans and share prices.

On a more practical level, every organization performs a variety of activities and spends a significant amount of resources to ensure the integrity of the information it delivers to customers, partners, suppliers, employees and shareholders. While these activities and resources reside in different parts of the organization, they are all aimed at ensuring the accuracy, consistency and reliability of information and information systems.

They may be viewed as part of an "Information integrity space", the dimensions of which may include activities related to: 
- Prevention, monitoring, detection, verification and correction of information errors;

- Security, audit and control;

- Protection against corruption of information due to accidental failures or deliberate fraud;

- Data scrubbing and cleansing in the creation of data warehouses;

- Design, development, operation, use and maintenance of information systems;

- Conversion of existing systems due to mergers, acquisitions and consolidations;

- Modification of existing systems to accommodate changes such as new legislation or new technology;

- Information integrity requirements of specific industries such as banking, finance, telecommunications, engineering, transportation, defense, etc.

Because different functional areas within an organization perform these activities, each addresses information integrity as an isolated, unique challenge, even though the underlying problems are inter-related and could be solved more effectively through an enterprise-wide approach. Consequently, many organizations are paying an inordinately high price for the level of information integrity they are able to achieve.

\section{COSTS OF INFORMATION INTEGRITY}

Most organizations have systems and procedures to verify the integrity of information they receive from both external and internal sources. These systems and procedures are usually custom-developed, unique to each organization and very labor-intensive. Many information integrity related activities are performed in all parts of the organization, and thus are often duplicated.

Although there are no readily available studies about the total cost of information integrity within an enterprise, many executives and industry experts concur that such costs would, as a conservative estimate, range from 1 percent to 5 percent of revenue. Effective enterprise- and industry-wide approaches to information integrity using standards, best practices and innovative technology would significantly reduce this cost. 


\section{INFORMATION INTEGRITY RISKS}

Many software vendors and information technology specialists claim their products and systems are not susceptible to information errors. However, all organizations are increasingly vulnerable to errors caused by information integrity failures, simply because such failures are primarily due to factors outside their systems and beyond their control. These exogenous factors include:

- Change: organizational structure, legislation, personnel, hardware, software, etc.;

- Complexity: the number and variety of components and interfaces; the volume and speed of information processing;

- Communication: the risk of duplicate, missed or partial transmission or receipt of information;

- Conversion: data consolidation, decomposition or transformation;

- Corruption: accidental failures and deliberate fraud.

When information integrity failures do occur, they can be enormously expensive, embarrassing and sometimes fatal. An enterprise-wide information integrity architecture, which minimizes an organization's exposure to the aforementioned exogenous factors, could effectively manage this risk.

\section{VALUE OF INFORMATION INTEGRITY}

Information integrity can create significant added value to organizations, especially those:

- Whose primary product is information;

- Whose core business processes are driven by information; or

- Who use their information base to develop new products, enter new markets or launch other strategic initiatives.

Organizations can realize this added value by proactively ensuring the integrity of the information about customers, markets, products and processes over a sufficient span of time.

\section{TECHNOLOGY EVOLUTION}

The rapid evolution of technology has steadily increased the impact and importance of information integrity, while at the same time making it 
possible to implement sophisticated solutions for achieving information integrity.

Stand-alone mainframe systems, for the first time, made it possible to automate the validation of input data and the programming of internal controls to verify the integrity of the processing logic. However, the implementation of the validation and controls was often ad hoc, arbitrary and ineffective. Many systems failed or produced erroneous results, and therefore required frequent reruns. These failures, however, did not always cause serious business disruptions, because systems had enough slack in the batch processing "window" to allow errors to be corrected.

The implementation of online systems for customer service shrank the batch processing window and made information integrity failures immediately visible to customers. This led to the implementation of automated balancing and reconciliation of the batch processing outputs. Despite these controls, the complexity of the online systems- and the lack of continuity between batch and online controls - exacerbated information integrity problems.

The introduction of client/server architectures further added to the complexity of information systems by increasing the number of hardware / software components and requisite interfaces. Distributed computing also increased the volume and frequency of data communication, thereby introducing the potential for duplicate, incomplete, missing or delayed data transmissions. The development of data warehouses, because it entails the conversion and consolidation of data from many disparate systems, has also been fraught with information integrity issues.

\section{RECENT TRENDS}

Several recent trends in information technology and the business environment dramatically intensify the urgency of finding radically different approaches and solutions to information integrity. These include:

- Information explosion;

- Application integration;

- Zero latency enterprise. 


\subsection{Information explosion}

Business, government and society are rapidly becoming informationdriven, with more and more of their functions based on information about customers, suppliers, employees, communities, citizens, products, capital and everything else. At the same time, we no longer can physically observe, measure or verify the name, address or other information about a customer; the financial capability of a supplier; or the amount of money in the bank. Instead, we manage our assets and deploy our resources based on the information we receive from our systems, databases or third-party resources. The Internet has dramatically multiplied the number of such third-party resources, as well as the amount of information available from each source. Since all this information is subject to the same types of errors and information integrity risks that were discussed earlier, we are forced to deal with massive amounts of information which, at best, is suspect.

\subsection{Application integration}

As organizations rapidly ramp up their presence on the World Wide Web and exponentially grow their e-commerce business, they quickly realize that their front-office systems must be integrated with both their back-office and distributed departmental systems. This forces them to undertake major application integration projects to provide straight-through processing for most of their mission-critical applications. These projects will reveal the serious information integrity deficiencies in their legacy applications and databases.

\subsection{Zero latency enterprise}

The net result of application integration will be to completely eliminate the batch cycles that have been characteristic of the information systems in every organization. Instead, customer's order or the supplier's invoice will be automatically processed the moment it is presented to the system, without any human intervention or verification, thus creating zero latency within the enterprise. Zero latency enterprises will have zero tolerance for information integrity errors.

\section{CONCLUSION}

Information integrity is a pervasive universal issue, which impacts business, government and society in profound ways in this electronic 
information age. Today, the knowledge and understanding about information integrity is rudimentary, fragmented and insufficient. Because of the lack of industry standards and best practices, we pay an inordinately high price for the current level of information integrity in our organizations. The recent trends in information technology and business dramatically heighten the seriousness and urgency of finding radical, different approaches and solutions for achieving information integrity.

Information integrity has the potential for becoming a new discipline, a new science, even a new industry, very much like the environmental science and industry, which emerged as a result of society's concerns about the quality of air, water and the earth.

Many of the answers to information integrity issues already exist in various other disciplines such as robotics, aeronautics and statistics. Similarly, much can be learned from the practices of other, more mature industries such as communication, transportation, utilities and even food.

The emergence of the new information integrity science, technology and industry will require the crafting and communication of clear, compelling and consistent messages about the pervasive and critical nature of information integrity, and the formation of a coalition of academia, thought leaders, professionals, practitioners and organizations interested in information integrity.

It will entail a broad spectrum of research, education, technology, standards, products and services. It is an opportunity for the champions of quality to make a difference. 